\title{
SENSIBILIDADE E ESPECIFICIDADE DA HISTEROSSONOGRAFIA NAS AFECÇÕES ENDOMETRIAIS EM MULHERES ASSINTOMÁTICAS PÓS-MENOPAUSA
}

\author{
*Sonia Tamanaha, José Mendes Aldrighi, Roberto Euzébio dos Santos, Roberto Adelino Almeida Prado \\ Trabalho realizado no Departamento de Obstetrícia e Ginecologia da Irmandade da Santa \\ Casa de Misericórdia de São Paulo - Faculdade de Ciências Médicas da Santa Casa de São Paulo
}

RESUMO - OBjetIVOs. I) Estimar sensibilidade e especificidade da histerossonografia nas afecções endometriais, utilizando-se como padrão-ouro a histeroscopia diagnóstica; 2) comparar concordância entre a ultra-sonografia, a histerossonografia e a histeroscopia através do índice de Kappa (K).

Métodos. Foram estudadas $\mathbf{5 0}$ mulheres assintomáticas após menopausa, todas tinham suspeita de afecções endometriais pela ultra-sonografia transvaginal e, por isso, complementaram avaliação endometrial com a histerossonografia, a histeroscopia diagnóstica e a biópsia orientada. Para comparaçáo dos resultados entre esses exames utilizou-se índice de Kappa.

Resultados. Afecções endometriais mais freqüentes: pólipos $(58 \%)$, sinéquias $(20 \%)$, mioma submucoso $(12 \%)$ e espessamento endometrial (6\%). Cavidade normal (4\%) dos exames histeroscópicos. A sensibilidade da histerossonografia para diagnóstico de pólipo foi de 89,7\%; a especificidade de $81 \%$ e o (K) de $71,1 \%$. Para sinéquia a sensibilidade foi de $80 \%$, a especificidade $100 \%$ e o (K) de 86,5\%; para mioma submucoso: a sensibilidade foi de $83,3 \%$; a especificidade de $97,7 \%$ e $\circ(\mathrm{K})$ de $81,1 \%$ e para espessamento endometrial a sensibilidade foi de $33,3 \%$, a especificidade de $89,4 \%$ e o (K) del5,5\%.

CONCLUSÖEs. A histerossonografia apresentou ótima concordância com a histeroscopia para sinéquias e miomas submucosos; boa concordância para pólipo e péssima concordância para espessamentos endometriais. Revelou-se, também, tratar-se de método simples, eficiente e que pode ser utilizado para a avaliação da cavidade uterina em mulheres após menopausa.

Unitermos: Histerossonografia. Pós-menopausa.

\section{INTRODUÇÃO}

As alterações demográficas constatadas nas últimas décadas' motivaram mudanças nas estratégias de vigilância adotadas como medidas preventivas ${ }^{2}$. Nesse contexto, o climatério merece atenção especial por ser um período caracterizado pela maior prevalência de afecções, dentre as quais destacam-se as endometriais ${ }^{3}$.

O desenvolvimento de métodos que possam rastrear afecções precursoras de neoplasias malignas é de grande interesse; o método diagnóstico ideal para esse objetivo deve ser eficiente, seguro, prático, de baixo custo e acessível $\left.\right|^{4,5}$.

Após a menopausa, o endométrio, em decorrência da falta de atividade estrogênica, apresenta-se inativo, mostrando histologicamente pequenas glândulas e o estroma denso, caracterizando o endométrio atrófico 6 .

Atrofia endometrial representa $\mathrm{O}$ achado histológico mais comum em mulheres após a

*Correspondência: Rua Frei Caneca, 444 - apto. 113 CEP: 01307-000 - Consolação - São Paulo - SP Fone: (11) 3255-1587 - Fax: (11) 3253-5766 E-mail: sonia.tamanaha@ig.com.br menopausa, apesar disso, a mucosa uterina continua responsiva aos estímulos esteroídicos endógenos ou exógenos, podendo sediar alterações proliferativas, hiperplásicas, bem como as neoplasias malignas?

Shipley III et al. ${ }^{8}$ mencionaram que a ultrasonografia transvaginal apresenta sensibilidade de $80 \%$ no rastreamento de anormalidades endometriais, permitindo dispensar a realização de investigação invasiva da cavidade uterina nos casos em que se identificam endométrios homogêneos, regulares e com espessuras $=5 \mathrm{~mm}^{9}$.

Apesar da facilidade de observação da espessura endometrial e da sua regularidade pela ultra-sonografia transvaginal, é difícil a constatação de aderências intra-uterinas, a diferenciação entre mioma submucoso e pólipo endometrial, a distinção entre endométrio proliferativo e hiperplasia endometrial ${ }^{|0,1|}$ e a mensuração da espessura endometrial, quando os nódulos miomatosos distorcem os limites da cavidade uterina' ${ }^{12}$.

A histeroscopia, por sua vez, permite não só o diagnóstico, mas também a terapêutica de inúmeras afecções, daí ser reconhecida como método de excelência na avaliação endometrial $^{13}$. Entretanto, seu custo, a necessidade de treinamento qualificado e o tempo dispensado na esterelização do equipamento não justificam seu emprego rotineiro em todas as mulheres assintomáticas ${ }^{14}$.

Assim, visando obter metodologia de avaliação endometrial efetiva, prática e de baixo custo desenvolveu-se a histerossonografia ${ }^{15,16}$. O presente estudo tem como objetivo quantificar a acurácia da histerossonografia no diagnóstico de mulheres assintomáticas após a menopausa.

\section{Métodos}

O projeto deste trabalho foi aprovado pela Comissão Científica do Departamento de Ginecologia e Obstetrícia da Santa Casa de São Paulo e pelo Comitê de Ética em Pesquisa da Irmandade de Misericórdia da Santa Casa de São Paulo.

Após a assinatura do Termo de Consentimento Pós-informado, foram incluídas no estudo 50 mulheres assintomáticas após a menopausa, entre 46 e 80 anos (média de 6I , I anos). Todas apresentavam anormalidades ao exame ultra-sonográfico transvaginal. O endométrio foi considerado anormal nas seguintes situações: 
Tabela I - Distribuição cruzada da ultra-sonografia transvaginal e histeroscopia em 50 mulheres assintomáticas após a menopausa

\begin{tabular}{|c|c|c|c|c|c|c|}
\hline \multirow[b]{2}{*}{$\begin{array}{l}\text { Resultados da ultra-sonografia } \\
\text { transvaginal }\end{array}$} & \multicolumn{6}{|c|}{ Resultadoshisteroscópicos } \\
\hline & $\begin{array}{l}\text { Cavidade } \\
\text { normal }\end{array}$ & Sinéquia & Pólipo & $\begin{array}{l}\text { Espessamento } \\
\text { endometrial }\end{array}$ & $\begin{array}{c}\text { Mioma } \\
\text { submucoso }\end{array}$ & Total \\
\hline $\begin{array}{l}\text { Ecoirregular } \\
\text { Pólipo } \\
\text { Espessamentoendometrial } \\
\text { Miomasubmucoso } \\
\text { Coleçãolíquida } \\
\text { Total }\end{array}$ & $\begin{array}{l}\text { I(50\%) } \\
\text { I(50\%) } \\
2(100 \%)\end{array}$ & $\begin{array}{l}3(30 \%) \\
1(10 \%) \\
5(50 \%) \\
1(10 \%) \\
10(100 \%)\end{array}$ & $\begin{array}{c}I(3,4) \\
7(24,1 \%) \\
20(69 \%) \\
1(3,4 \%) \\
29(100 \%)\end{array}$ & $\begin{array}{l}2(66,7 \%) \\
1(33,3 \%)\end{array}$ & $\begin{array}{l}2(33,3 \%) \\
4(66,7 \%) \\
6(100 \%)\end{array}$ & $\begin{array}{c}4(8 \%) \\
10(20 \%) \\
29(58 \%) \\
5(10 \%) \\
2(4 \%) \\
50(100 \%)\end{array}$ \\
\hline
\end{tabular}

Taxa de concordância dos resultados da ultra-sonografia transvaginal e histeroscopia: $24 \%$

espessura endometrial $\geq 5 \mathrm{~mm}$; espessamento focal ou interrupção do eco endometrial de forma e extensão variadas; ausência de linha ecodensa central; heterogeneidade focal ou difusa da ecotextura endometrial; presença de coleção líquida e espessura endometrial $>3 \mathrm{~mm}$ ao seu redor ou espessamento focal ou irregularidade do eco endometrial.

Entre os critérios de exclusão destacam-se as condições que impediram a realização da histerossonografia e/ou histeroscopia ambulatorial, como: a estenose vaginal e/ou estenose cervical, o desconforto acentuado durante os exames, a infecção vaginal ou cervical; o uso de terapia de reposição hormonal nos últimos 12 meses e a presença de tumores ovarianos.

As ultra-sonografias transvaginais foram realizadas após esvaziamento vesical, utilizando-se do transdutor endocavitário (HITACHI EUB-405, 6.5 MHz, JAPAN).

$\mathrm{Na}$ avaliação da cavidade uterina inclúram-se: a espessura, homogeneidade endometrial, linearidade e continuidade da linha de demarcação entre o endométrio e miométrio ${ }^{17}$. $O$ endométrio era medido no plano longitudinal (incluindo as duas interfaces), no seu segmento mais espesso, no sentido anteroposterior e foi considerado espesso quando os valores obtidos mostraram-se $=5 \mathrm{~mm}^{18}$.

A histerossonografia era indicada quando o exame ultra-sonográfico constatava espessamento endometrial, irregularidade da linha de demarcação entre o endométrio e miométrio, ou quando suspeitou-se de outras afecções da cavidade uterina.

A histerossonografia era realizada consoante a seguinte seqüência: exposição do colo uterino com o auxílio do espéculo vaginal de Collins, assepsia realizada com polvidine tópico (PVPI), introdução de um cateter infantil na cavidade uterina (I-Cath B-D, intravenoso, com guia metálico, comprimento de $30 \mathrm{~cm}$ e diâmetro de $17 \mathrm{G}$ ), remoção do espéculo e posicionamento do transdutor transvaginal (previamente revestido com condom) posteriormente ao cateter.

Pelo cateter instilava-se de $3 \mathrm{ml}$ a $10 \mathrm{ml}$ de solução salina, objetivando-se a distensão e a avaliação ultra-sonográfica da cavidade uterina, tanto no plano longitudinal como no transversal do colo até o fundo uterino. As imagens foram interpretadas de acordo com os critérios de Parsons \& Lense ${ }^{19}$.

Após a histerossonografia, as mulheres eram encaminhadas para a avaliação histeroscópica ambulatorial, sem anestesia, utilizando-se do histeroinsuflador de dióxido de carbono (Karl-Storz-ALEMANHA 26026 U) e o histeroscópio (Endoview-BRASIL) rígido, com angulação de $30^{\circ}$ e 4 mm de diâmetro. Os achados histeroscópicos foram classificados segundo os critérios de Hamou EJ ${ }^{20}$.

As biópsias endometriais foram efetuadas por meio de pinça saca-bocados, sendo que todas as amostras obtidas foram encaminhadas para o exame histopatológico.

Para a avaliação da concordância da histerossonografia com a histeroscopia diagnóstica foram estimadas a sensibilidade, especificidade, os valores preditivos (negativo e positivo) e o índice de Kappa. Esse índice quantifica a concordância entre dois métodos realizados nos mesmos indivíduos, sendo assim interpretados: $81 \%$ a $100 \%$ (ótima); $61 \%$ a $80 \%$ (boa); $41 \%$ a $60 \%$ (regular); $21 \%$ a $40 \%$ (ruim) e menor do que 20\% (péssima)

\section{Resultados}

O estudo incluiu, inicialmente, 75 mulheres. Entretanto, foram excluídas 19 mulheres por estenoses do canal cervical e seis por abandonarem a investigação após a intrans- ponibilidade cervical durante a histerossonografia; assim, a casuística totalizou 50 muIheres assintomáticas após a menopausa, que foram submetidas à ultra-sonografia transvaginal, à histerossonografia, à histeroscopia e ao exame histopatológico.

Ao exame ultra-sonográico, 38 mulheres (76\%) apresentaram espessura endometrial = $5 \mathrm{~mm}$ e nove ( $18 \%$ ) apresentaram espessura endometrial $<5 \mathrm{~mm}$, mas quatro delas tinham ecos endometriais irregulares e/ou heterogêneos, dois foram os casos de mulheres com coleção líquiida na cavidade uterina associada à irregularidade do eco endometrial, duas com imagens sugestivas de pólipos e uma com mioma submucoso.

A espessura endometrial foi aferida pela ultra-sonografia transvaginal em 47 (94\%) das 50 mulheres. A menor espessura endometrial foi de $2,5 \mathrm{~mm}$ e a maior, $17,6 \mathrm{~mm}$, sendo que a média e o desvio-padrão foram, respectivamente, $8,4 \mathrm{~mm}$ e $3,5 \mathrm{~mm}$.

Em três mulheres (6\%), a espessura endometrial não foi mensurada, devido à distorção significativa dos limites do eco endometrial, provocada pela presença de nódulos miomatosos.

O espessamento endometrial foi o achado mais freqüente da ultra-sonografia transvaginal, 29 casos (58\%), seguido por dez casos de pólipo (20\%), cinco casos de mioma submucoso ( $10 \%)$, quatro de eco endometrial irregular (8\%) e dois casos de coleção líquida na cavidade com irregularidade do eco endometrial (4\%).

Dos 29 espessamentos endometriais, um caso foi confirmado pela histeroscopia e 20 casos foram diagnosticados como pólipos (Tabela I).

A taxa de concordância dos resultados da ultra-sonografia transvaginal e da histeroscopia foi de $24 \%$. 
Tabela 2 - Distribuição cruzada da histerossonografia e histeroscopia em 50 mulheres assintomáticas após a menopausa

\begin{tabular}{|c|c|c|c|c|c|c|}
\hline \multirow[b]{2}{*}{$\begin{array}{l}\text { Resultados da } \\
\text { histerossonografia }\end{array}$} & \multicolumn{6}{|c|}{ Resultados histeroscópicos } \\
\hline & $\begin{array}{c}\text { Cavidade } \\
\text { normal }\end{array}$ & Sinéquia & Pólipo & $\begin{array}{c}\text { Espessamento } \\
\text { endometrial }\end{array}$ & $\begin{array}{c}\text { Mioma } \\
\text { submucoso }\end{array}$ & Total \\
\hline Pólipo & | (50\%) & $1(10 \%)$ & $26(89,7 \%)$ & $2(66,7 \%)$ & & $30(60 \%)$ \\
\hline Espessamento endometrial & $\mid(50 \%)$ & $1(10 \%)$ & $2(6,9 \%)$ & I $(33,3 \%)$ & $\mid(16,7 \%)$ & $6(12 \%)$ \\
\hline Miomasubmucoso & & & I (3,4\%) & & $5(83,3 \%)$ & $6(12 \%)$ \\
\hline
\end{tabular}

Taxa de concordância dos resultados da histerossonografia e histeroscopia: $80 \%$

Tabela 3 - Distribuição cruzada dos resultados histopatológicos e histeroscópicos em 50 mulheres assintomáticas após a menopausa

\begin{tabular}{|c|c|c|c|c|c|c|}
\hline \multirow[b]{2}{*}{$\begin{array}{l}\text { Resultados } \\
\text { histopatológicos }\end{array}$} & \multicolumn{6}{|c|}{ Resultadoshisteroscópicos } \\
\hline & $\begin{array}{c}\text { Cavidade } \\
\text { normal }\end{array}$ & Sinéquia & Pólipo & $\begin{array}{l}\text { Espessamento } \\
\text { endometrial }\end{array}$ & $\begin{array}{c}\text { Mioma } \\
\text { submucoso }\end{array}$ & Total \\
\hline
\end{tabular}

Taxa de concordância dos resultados histopatológicos e histeroscópicos: 36\%

Tabela 4 - Sensibilidade, especificidade, valor preditivo positivo (VPP) e valor preditivo negativo (VPN) e índice de Kappa

(K) da histerossonografia para as diferentes afecções endometriais

\begin{tabular}{|c|c|c|c|c|c|}
\hline Histerossonografia & Sensibilidade & Especificidade & VPP & VPN & $K$ \\
\hline Pólipo & 89,7 & 81,0 & 86,7 & 85,0 & 71,1 \\
\hline Sinéquia & 80,0 & 100,0 & 100,0 & 95,2 & 86,5 \\
\hline Espessamento endometrial & 33,3 & 89,4 & 16,7 & 95,5 & $\mid 5,5$ \\
\hline Miomasubmucoso & 83,3 & 97,7 & 83,3 & 97,7 & 81,1 \\
\hline
\end{tabular}

Nota: valores expressos em porcentagem

A histerossonografia demostrou: 30 pólipos (60\%), oito sinéquias intra-uterinas (16\%), seis espessamentos endometriais (12\%) e seis miomas submucosos (12\%). Quando a histerossonografia foi comparada à histeroscopia, para o diagnóstico das afecções da cavidade uterina, a taxa de concordância dos resultados foi de $80 \%$ (Tabela 2).

A comparação entre os resultados histopatológicos e os histeroscópicos mostrou taxa de concordância de 36\% (Tabela 3).

Os resultados quanto à sensibilidade, à especificidade e ao índice de Kappa para a histerossonografia estão apresentados na Tabela 4.

Analisando-se os índices de Kappa para o diagnóstico de pólipo, observa-se que a histerossonografia revelou boa concordância ( $K=71$, I 5 \%) com a investigação histeros- cópica. Para o diagnóstico de sinéquia, a histerossonografia mostrou ótima concordância ( $K=86,5 \%$ ). $O$ índice de Kappa de 15,5\% da histerossonografia para espessamento endometrial foi interpretado como de péssima concordância com os resultados histeroscópicos. Índice de Kappa de 81,1\% para o mioma submucoso classificou o método histerossonográfico como de ótima concordância com a histeroscopia.

\section{Discussão}

Com a introdução dos transdutores transvaginais para a realização dos exames ultra-sonográficos, a espessura endometrial em mulheres após a menopausa tem sido comparada com os resultados histológicos. Isso tem propiciado uma redução expressiva do número de procedimentos invasivos da cavidade uterina para o diagnóstico das anormalidades endometriais ${ }^{22}$.

A literatura assinala boa sensibilidade e especificidade da ultra-sonografia transvaginal no diagnóstico das patologias endometriais. De fato, Nasri \& Coast $^{18}$, estudando 90 mulheres após a menopausa, 63 com sangramento uterino e 27 com ginecopatias cirúrgicas, e correlacionando com a histologia endometrial de material proveniente das peças cirúrgicas ou da curetagem uterina, constataram sensibilidade de 91\% e especificidade de 100\%, quando o limite de corte para a espessura endometrial foi de $5 \mathrm{~mm}$.

Em mulheres sintomáticas ou em uso de terapia de reposição hormonal, o endométrio tem sido exaustivamente avaliado pela ultrasonografia transvaginal como método de triagem para anormalidades. No entanto, poucos 
TAMANAHA S ET AL.

são os estudos sobre os achados da ultrasonografia transvaginal em mulheres assintomáticas após a menopausa e não usuárias de terapia de reposição hormonal23.

O grupo de estudo foi constituido por mulheres assintomáticas após a menopausa, $38 \mathrm{com}$ eco endometrial $=5 \mathrm{~mm}(76 \%) \mathrm{e}$ nove com espessuras endometriais $<5 \mathrm{~mm}$ (। 8\%), mas quatro apresentaram ecos irregulares e ou heterogêneos; duas, coleção líquida na cavidade e irregularidade do eco endometrial; duas, imagem sugestiva de pólipo; e uma, mioma submucoso.

A média da espessura endometrial obtida foi de $8,4 \mathrm{~mm}$, sugerindo-se endométrios ativos. Apesar da falência estrogênica após a menopausa, o endométrio pode responder a estímulos hormonais, dependendo da concentração de aromatases no tecido adiposo, que permite a conversão periférica de androgênicos em estrona, explicando-se assim as alterações proliferativas, hiperplásicas e, eventualmente, carcinomatosas ${ }^{24}$.

É importante salientar que não se pretendeu substituir a histologia pela interpretação dos métodos de diagnóstico por imagens, mas sim nortear a abordagem da afecção identificada.

Obteve-se taxa de concordância entre a ultra-sonografia transvaginal e histeroscopia de $24 \%$. Torna-se importante salientar que os exames ultra-sonográficos não asseguraram diagnóstico exato da alteração endometrial, mas assinalaram os casos a serem investigados por outras técnicas ${ }^{25}$. Das 50 mulheres previamente selecionadas pela ultra-sonografia, a histeroscopia demonstrou a presença de afecção endometrial em 48 delas (96\%) e, em duas (4\%), a cavidade mostrou-se normal.

Nesse estudo, os resultados discordantes entre a ultra-sonografia e histeroscopia ocorreram com maior freqüência no espessamento endometrial. Por isso, esse achado ultra-sonográfico foi considerado inespecífico, pois decorre de vários substratos histológicos, como: pólipos, miomas submucosos, sinéquias intra-uterinas e hiperplasias. Essa dificuldade na diferenciação dos processos que expandem a cavidade uterina também foi apontada por Fleischer et al. ${ }^{23}$.

Duas mulheres do estudo mostraram coleção líquida na cavidade uterina e nenhuma se correlacionou com câncer de endométrio. Esses resultados diferem dos obtidos por
Breckenridge et al. ${ }^{26}$ que encontraram 94, $12 \%$ de associação entre fluido intra-cavitário e câncer de endométrio. No entanto, aquele grupo de mulheres após a menopausa apresentava sintomas como dor, sangramento genital ou massas pélvicas e estenose cervical em 65\% dos casos.

Consoante Goldstein ${ }^{27}$, mulheres após a menopausa podem apresentar endométrio atrófico associado à estenose cervical e à coleção líquida na cavidade, por isso dispensaria biópsias se a espessura endometrial ao redor da coleção fosse $<3 \mathrm{~mm}$.

Encontrou-se nesse estudo a taxa de concordância de $80 \%$ entre os resultados da histerossonografia e da histeroscopia.

Wolman et al. ${ }^{14}$ encontraram sensibilidade de $86 \%$ e especificidade de $100 \%$ para a histerossonografia, estudando 50 mulheres assintomáticas após a menopausa com espessamentos endometriais (> $15 \mathrm{~mm}$ ).

Parsons \& Lense ${ }^{19}$ avaliaram 39 pacientes antes e após a menopausa pela histerossonografia e concluíram que se trata de uma excelente técnica para discriminação entre afecção intracavitária, intramural e processos difusos.

Em nosso estudo, a sensibilidade da histerossonografia foi de 89,7\% para o diagnóstico de pólipo. Para espessamento endometrial, o método apresentou sensibilidade de $33,3 \%$ e especificidade de $89,4 \%$.

Nenhuma sinéquia foi diagnosticada ao exame ultra-sonográfico, enquanto que a histerossonografia demonstrou a presença de oito das dez sinéquias ratificadas pela histeroscopia.

Outra grande contribuição da infusão de solução salina na cavidade uterina é visibilizar a espessura endometrial quando na presença de miomas submucosos ou miomas intramurais, que distorcem os limites do eco endometrial e prejudicam a sua mensuração ${ }^{17}$. Em nosso estudo, a sensibilidade da histerossonografia para mioma submucoso foi de $83,3 \%$.

No entanto, a intransponibilidade cervical para realização dos vários métodos endouterinos após a menopausa, em regime ambulatorial, oscila em torno de 10\%, sendo maior com o avançar do tempo após a menopausa ${ }^{28}$. Durante a seleção do nosso grupo de estudo, observamos a presença de $21,33 \%$ de estenoses do canal cervical que inviabilizaram a realização da histerossonografia.
Os riscos da histerossonografia são pequenos. Dor e desconforto são minimizados pela pequena quantidade de fluido instilado, ou seja, de $3 \mathrm{ml} \mathrm{a} 10 \mathrm{ml}$ de solução salina. Não constatamos perfurações uterinas, nem infecções pélvicas como citado na literaturara-3! Outra temível, porém teórica complicação é o risco de disseminação de neoplasia maligna endometrial. Isso poderia ocorrer se fosse utilizado fluxo de alta pressão durante 0 procedimento, que acarretaria passagem de solução salina para as tubas uterinas ${ }^{29}$. $\mathrm{O}$ uso de cateter de única via, por nós preconizado, pode reduzir esse risco, pois facilita o refluxo pelo canal cervical, antes do extravasamento tubário.

Atualmente, a histeroscopia é considerada o melhor método para avaliar a cavidade endometrial e apresenta acurácia diagnóstica de $95 \%$, sensibilidade de $91 \%$ a $98 \%$ e especificidade de $95,5 \%$ a $100 \%{ }^{13}$. Quando analisada individualmente, identifica tanto patologias endometriais benignas como malignas, com $20 \%$ de falso-positivos e nenhum falso-negativo ${ }^{30}$.

No entanto, não se justifica sua indicação como método de rastreamento em uma população assintomática, principalmente em serviços com grande demanda de pacientes ${ }^{14}$. Outro ponto a ser ressaltado é que a histeroscopia ambulatorial não permite a realização de biópsias sob visão direta, podendo omitir casos de alterações endometriais ${ }^{32}$.

De fato, obtivemos $40 \%$ de material considerado insuficiente para análise histopatológica. Esse alto índice de insucesso pode ter ocorrido pelas biópsias realizadas sem visão histeroscópica direta e também pela dificuldade em abordar as lesões pediculadas. Considerando-se essas limitações técnicas, em algumas situações, a cirurgia histeroscópica poderia ser otimizada, dispensando-se a histeroscopia ambulatorial.

Para um exame ser considerado útil, ele deve sobreviver a uma série de quesitos rigorosos, que avaliam sua reprodutibilidade, acurácia e factibilidade, bem como seus efeitos nas decisões clínicas e nos desfechos. A aplicação do exame passa a ser valorizada quando aumenta a probabilidade de um diagnóstico correto ou quando ele é mais seguro ou menos dispendioso do que os já existentes ${ }^{33}$.

O cálculo do tamanho amostral realizado durante o delineamento desse estudo foi 
considerado representativo do grupo de mulheres após a menopausa matriculadas na Clínica de Ginecologia Endócrina e Climatério da Santa Casa de São Paulo e consideramos que esses resultados podem ser utilizados para análises individuais.

\section{Conclusões}

Neste estudo, a histerossonografia apresentou ótima concordância com a histeroscopia para sinéquias e miomas submucosos; boa concordância para pólipo e péssima concordância para espessamentos endometriais. Revelou-se, também, tratar-se de método simples, eficiente e que pode ser utilizado para a avaliação da cavidade uterina em mulheres após menopausa.

\section{Agradecimento}

Agradecemos ao NAP-SC (Núcleo de Apoio à Publicação da Faculdade de Ciências Médicas da Santa Casa de São Paulo) pelo suporte técnicocientífico à publicação.

\section{Conflito de interesse: não há.}

\section{SUMMARY \\ Sensitivity and SPECIFICITY OF HYSTE- ROSONOGRAPHY IN ENDOMETRIAL ABNOR- MALITIES IN ASYMPTOMATIC POSTME- NOPAUSAL WOMEN}

BACKGROUND. To estimate sensitivity and specificity of hysterosonography for diagnosis of endometrial cavity abnormalities. The goldstandard was hysteroscopy; to compare the agreement between ultrasonographic, hysterosonographic and hysteroscopic findings using the KIA (Kappa Index Agreement).

METHODS, Fifty asymptomatic postmenopausal women that had a suspicion of endometrial abnormalities based upon transvaginal ultrasonography were studied. Hysterosonography, diagnostic hysteroscopy and oriented biopsy were performed and the Kia was used to compare results.

RESULTS. The most frequent abnormalities were polyps (58\%), synechiae (20\%), submucous myoma (12\%) and endometrial thickening (6\%). The uterine cavity was considered normal in $4 \%$ of the evaluations by hysteroscopy.

The sensitivity of hysterosonography to diagnose polyps was of $89.7 \%$, the specificity of $81.0 \%$ and the KIA of $71.1 \%$. For synechia sensitivity of hysterosonography was of $80 \%$, specificity of $100 \%$ and the KIA of $86.5 \%$; for submucous myoma sensitivity was of $83.3 \%$; specificity of $97.7 \%$ and the KIA of $81.1 \%$, and for endometrial thickening, sensitivity was of $33.3 \%$, specificity of $89.4 \%$ and the KIA of $15.5 \%$.

ConClusion. Hysterosonographyshowed very good agreement with hysteroscopy for the diagnosis of synechiae and submucous myomas; good agreement for polyps and poor agreement for endometrial thickening. Based upon this data hysterosonography may be deemed a simple, efficient, and accurate method for the evaluation of the uterine cavity in the postmenopausal period. [Rev Assoc Med Bras 2004; 50(4): 427-32]

KEY WORDS: Hysterosonography. Postmenopause.

\section{REFERÊNCIAS}

I. IBGE. Instituto Brasileiro de Geografia e Estatística. Expectativa de vida do brasileiro sobe 2,6 em uma década. O Brasil no final do século 20. [Folha Online] [citado 2002 nov]. Disponível em: htttp://wwwl.uol.com.br/ folha/cotidiano/200 I-brasil-seculo_20.shtml.

2. Aldrighi JM, Pires ALR. Climatério/TRH. Reprod Clim 200 I; I 6:24-30

3. Sociedade Brasileira de Cancerologia. Endométrio. [citado 2002 out]. Disponível em: http:/ /www.sbcancer.org.br/htmls/artigos.htm.

4. Wehba S. Teste de progesterona para detecção de lesões hiperplásicas do endométrio em mulheres na pós-menopausa [tese]. São Paulo: Escola Paulista de Medicina; 1988.

5. Cohen MA, Sauer MV, Keltz M, Lindheim S.R. Utilizing routine sonohysterography to detect intrauterine pathology before initiating hormone replacement therapy. Menopause 1999; 6:68-70

6. Crum CP. Aparelho genital feminino. In: Contran RS, editor. Robbins patologia estrutural e funcional. $6^{\mathrm{a}} \mathrm{ed}$. Rio de Janeiro: Guanabara-Koogan; 2000. p.929-78.

7. Gonçalves WJ, Bortoletto CCR, Stávale JN, Haidar MA, Simões RD, Baracat EC. Correlação entre a ultra-sonografia transvaginal e o exame anatomopatológico do endométrio de mulheres assintomáticas na pósmenopausa. Estudo prospectivo de 300 casos. J Bras Ginecol 1995; 105:89-96.

8. Shipley CF III, Simmons CL, Nelson GH. Comparison of transvaginal sonography with endometrial biopsy in asymptomatic postmenopausal women. J Ultrasound Med 1994; 13:99-104.

9. Goldstein SR. Use of ultrasonohysterography for triage of perimenopausal patients with unexplained uterine bleeding. Am J Obstet Gynecol 1994; 170:565-70.
10. Cullinan JA, Fleischer AC, Kepple DM, Arnold AL. Sonohysterography: a technique for endometrial evaluation. Radiographics 1995; | 5:50 | - | 4

II. Cicinelli E, Romano F, Anastasio PS, Blasi N, Parisi C. Sonohysterography versus hysteroscopy in the diagnosis of endouterine polyps. Gynecol Obstet Invest 1994;38:266-71.

12. Cicinelli E, Romano F, Anastasio PS, Blasi N, Parisi C, Galantino P. Transabdominal sonohysterography, transvaginal sonography, and hysteroscopy in the evaluation of submucous myomas. Obstet Gynecol 1995:85:42-7.

13. Gonçalves MAG, Piovesan C, Fedrizzi E, Cunha Filho JS, Melki RA, Poli M, et al. Histeroscopia diagnóstica na pós-menopausa. In: Donadio N, Albuquerque Neto LC, editors. Consenso Brasileiro em Videoendoscopia Ginecológica. Rio de Janeiro: Aché Laboratórios Farmacêuticos; 200 I. p. 332-5.

I4. Wolman I, Jaffa AJ, Hartoov J, Bar-Am A, David MP. Sensitivity and specificity of sonohysterography for the evaluation of the uterine cavity in perimenopausal patients. J Ultrasound Med 1996; 15:285-8.

15. Goldstein SR. Saline infusion sonohysterography. Clin Obstet Gynecol 1996; 39:248-58

16. Laughead MK, Stones LM. Clinical utility of saline solution infusion sonohysterography in a primary care obstetric-gynecologic practice. Am J Obstet Gynecol 1997: 176:1313-8.

17. Lev-Toaff AS. Sonohysterography: evaluation of endometrial and myometrial abnormalities. Semin Roentgenol 1996;31:288-98.

18. Nasri MN, Shepherd JH, Setchell ME, Lowe DG, Chard T. The role of vaginal scan in measurement of endometrial thickness in postmenopausal women. $\mathrm{Br}$ J Obstet. Gynaecol 1991;98:470-5.

19. Parsons AK, Lense JJ. Sonohysterography for endometrial abnormalities: preliminary results. J Clin Ultrasound 1993;2 1:87-95.

20. Hamou EJ. Hysteroscopy and microscopohysteroscopy: text and atlas. Norwalk: Appleton \& Lange; 1991.p.340.

21. Altman DG. Practical statistics for medical research. Londres: Chapman and Hall; 1991.

22. Granberg S, Wikland M, Karlsson B, Norstrom A, Friberg LG. Endometrial thickness as measured by endovaginal ultrasonography for identifying endometrial abnormality. Am J Obstet Gynecol 1991; 164:47-52.

23. Fleischer AC, Wheeler JE, Lindsay I, Hendrix SL, Grabill S, Kravitz B, et al. An assessment of the value of ultrasonographic screening for endometrial disease in postmenopausal women without symptoms. Am J Obstet Gynecol 200 I; | 84:70-5.

24. Giusa-Chiferi MG, Goncalves WJ, Baracat EC, Albuquerque Neto LC, Bortoletto CC, De Lima GR. Transvaginal ultrasound, uterine biopsy and hysteroscopy for postmenopausal bleeding. Int J Gynaecol Obstet 1996;55:39-44. 
TAMAnaha S ET AL.

25. Karlsson B, Granberg S, Wikland M, Ylostalo P, Torvid K, Marsal K, et al. Transvaginal ultrasonography of the endometrium in women with postmenopausal bleeding-a Nordic multicenter study. Am J Obstet Gynecol 1995; 172: 1488-94.

26. Breckenridge JW, Kurtz $A B$, Ritchie WG, Macht EL Jr. Postmenopausal uterine fluid collection: indicator of carcinoma. AJR Am J Roentgenol 1982; 139:529-34.

27. Goldstein SR. Postmenopausal endometrial fluid collections revisited: look at the doughnut rather than the role. Obstet Gynecol 1994:83:738-40.

28. Vuopala S, Kauppila A, Mikkonen M, Stenback F. Screening of asymptomatic postmenopausal women for gynecological malignancies, with special reference to endometrial sampling methods. Arch Gynecol 1982;231:119-27.

29. Dubinsky TJ, Parvey HR, Gormaz G, Maklad N. Transvaginal hysterosonography in the evaluation of small endoluminal masses. J Ultrasound Med 1995; 1 4: I-6.

30. Mencaglia L, Albuquerque Neto LC. Aspectos histeroscópicos do carcinoma endometrial. histeroscopia diagnóstica. Rio de Janeiro: MEDS; 2002. p. I55-66.

31. Izzo CR. Contribuição da histerossonografia no estudo da cavidade uterina [dissertação]. São Paulo: Faculdade de Medicina, Universidade de São Paulo; 2000.

32. Dubinsky T], Parvey R, Gormaz G, Curtis M, Maklad N. Transvaginal hysterosonography: comparison with biopsy in the evaluation of postmenopausal bleeding. J Ultrasound Med 1995: | 4:887-93.

33. Newman TB, Browner WS, Cummings SR Delineando estudos de testes médicos. In: HulleySB, Cummings SR, BrownerWS, Grady D, Hearst N, Newman TB, editors. Delineando a Pesquisa Clínica: Uma Abordagem epidemiológica. 2a ed. Porto Alegre: Artmed Editora; 2003. p.203-19.

Artigo recebido: 08/08/03

Aceito para publicação: 16/02/04 